\title{
Dificultades en la percatación rápida de incongruencias en el trastorno de aprendizaje procedimental: posible disfunción de la coherencia central
}

\author{
Nerea Crespo-Eguílaz, Juan Narbona
}

Introducción. El trastorno de aprendizaje procedimental, o trastorno de aprendizaje no verbal, afecta a la automatización de destrezas y de rutinas perceptivomotoras y cognitivas.

Objetivo. Profundizar en la disfunción neurocognitiva de este trastorno; en concreto, analizar las dificultades que experimentan al pasar del reconocimiento de los elementos de un conjunto a la comprensión del todo.

Desarrollo. La confrontación de informaciones visuales simultáneas y la percatación rápida de incongruencias son habilidades especialmente difíciles en niños con trastorno de aprendizaje procedimental.

Conclusiones. Estas dificultades pueden reflejar una disfunción de la coherencia central y explicar, en parte, el déficit en la adaptación de la conducta social que presentan estos niños.

Palabras clave. Aprendizaje no verbal. Aprendizaje procedimental. Coherencia central. Déficit de atención. Integración visuoespacial. Percatación de incongruencias.

\section{Introducción}

El trastorno de aprendizaje procedimental (TAP), o trastorno de aprendizaje no verbal [1], afecta a la automatización de destrezas perceptivomotoras y de rutinas cognitivas que, normalmente, se usan sin especial esfuerzo. Estas destrezas y rutinas se convierten, para los afectos del TAP, en procesos trabajosos que demandan un coste suplementario de la atención y de la memoria operativa, lo que va en detrimento de la comprensión rápida de situaciones en las que hay que manejar información de cierta complejidad $[1,2]$.

A consecuencia de esto, en el TAP se observa un trastorno del desarrollo de la coordinación, dificultad en los aprendizajes escolares, lentitud en los procesos y dificultad en la comprensión y en la adaptación de la conducta social. Es frecuente la asociación con el trastorno por déficit de atención/hiperactividad (TDAH), habitualmente bajo la forma con predominio de inatención $[1,2]$.

Se han documentado también en esta patología alteraciones en la integración visuoespacial: en el reconocimiento de los detalles, en el procesamiento simultáneo, en la organización visuoespacial y en la integración de las partes en un todo $[3,4]$.

Pretendemos analizar y profundizar en la disfunción neurocognitiva de este trastorno. Concretamen- te, revisamos aquí las dificultades que los escolares afectos de TAP experimentan en pasar del reconocimiento de los elementos de un conjunto al sentido (coherencia) o sinsentido del todo, y viceversa. La realización rápida de esta tarea, bajo el control del sistema ejecutivo, es fundamental en la conducta social y en los requerimientos de aprendizaje complejo. En este trabajo se realiza un metaanálisis de los hallazgos al respecto en la bibliografía y de nuestra propia experiencia clínica.

\section{Coherencia central y trastornos del desarrollo}

La coherencia central es una característica del procesamiento de la información que permite integrar la información diversa del contexto para elaborar representaciones significativas globales [5]; proporciona la coherencia perceptiva y conceptual en dominios tanto verbales como visuales [6]. Este concepto está relacionado con los tipos de procesamiento de la información bottom-up/top-down y con los estilos cognitivos clásicos de dependencia/ independencia de campo [7-9].

Las informaciones visuales se elaboran en dos amplios sistemas de integración tras alcanzar el lóbulo occipital: el sistema occipitoparietal (vía del
Unidad de Neurología Pediátrica. Departamento de Pediatría. Clínica Universidad de Navarra. Pamplona, Navarra, España.

Correspondencia: Dra. Nerea Crespo Eguílaz. Departamento de Pediatría. Clínica Universidad de Navarra. Avda. Pío XII, 36. E-31008 Pamplona (Navarra).

E-mail:

necrespo@unav.es

Financiación:

Fundación Fuentes Dutor

Aceptado tras revisión externa: 20.01.11.

Cómo citar este artículo: Crespo-Eguílaz N, Narbona J. Dificultades en la percatación rápida de incongruencias en el trastorno de aprendizaje procedimental: posible disfunción de la coherencia central. Rev Neurol 2011; 52 (Supl 1): S39-41. 
'dónde') y el sistema occipitotemporal (vía del 'qué') [10]; el primero identifica las relaciones espaciales y el movimiento de los objetos, mientras que el segundo reconoce los objetos accediendo a la memoria semántica. Estos sistemas están bien diferenciados, pero se relacionan mediante conexiones córtico-estriotalámicas y córtico-corticales a través del lóbulo frontal y los sistemas de memoria operativa y a largo plazo. Además, el reconocimiento de una imagen visual necesita de la interacción entre las dos vías visuales y de la confrontación con la memoria semántica, en la que intervienen el lóbulo frontal y el sistema hipocampo-tálamo-cortical $[11,12]$.

La teoría de la coherencia central de Uta Frith sugiere que, en los niños con autismo, una falta de coherencia central puede explicar algunos de los déficit no explicados por la teoría de la mente [13]. Tomando como base esta teoría, las personas con autismo tienen ventajas significativas en aquellas tareas en las que es útil un procesamiento preferente de las partes sobre el todo, pero manifiestan desventajas considerables en las que requieren una interpretación de los elementos individuales en función del contexto y del significado global. Un ejemplo interesante es el procesamiento de caras: no tienen problemas para procesar rasgos faciales, pero sí para reconocer la expresión emocional, donde es necesario un procesamiento global de los rasgos [5]. En la bibliografía, numerosas investigaciones hacen referencia al déficit de coherencia central en el autismo y en el síndrome de Asperger [14-16]. En la actualidad existe el debate de si el sesgo atencional hacia aspectos locales se debe a un mayor procesamiento perceptivo o es el resultado de una dificultad en la integración de la información [6].

La coherencia central nos permite captar lo esencial de una historia, identificar con facilidad lo relevante de lo superfluo en una situación, reconocer el sentido de palabras ambiguas según el contexto, etc. Por consiguiente, presentar una coherencia central débil significa tener un estilo cognitivo caracterizado por la tendencia al procesamiento de detalles, que implica darse cuenta de conexiones no habitualmente percibidas por otras personas, y por la dificultad para integrar la información en un todo coherente $[5,17]$. La débil coherencia central está relacionada con las alteraciones en algunas funciones ejecutivas, como, por ejemplo, la inhibición de respuestas incorrectas en función del contexto [18]. Las pruebas más utilizadas en la investigación se basan principalmente en tareas verbales $[15,19,20]$.

\section{Coherencia central en el trastorno de aprendizaje procedimental}

La disfunción de la coherencia central no es exclusiva del autismo y puede observarse también en sujetos con TAP o en el retraso mental, entre otros. En el ámbito escandinavo, Gillberg [21] indica que los niños con déficit de atención, del control motor y de la percepción manifiestan también un estilo cognitivo similar.

Tener coherencia central débil implica identificar los detalles de un relato o discurso pero sin comprenderlo en su conjunto, tener dificultades para describir los aspectos importantes de una escena o situación, proporcionar información que no viene al caso...; en definitiva, una dificultad para la comprensión contextual de las situaciones sociales así como para adaptar la conducta en ellas, como les sucede a los niños con TAP [1]. Además, la identificación de nuevas representaciones contribuye a que inviertan más tiempo en las tareas escolares que requieren de procesamiento simultáneo de la información [17].

En nuestra experiencia clínica [22] nos resultan útiles dos tareas de percepción visual que precisan, para su correcta comprensión, de la confrontación de informaciones visuales concurrentes y de una percatación rápida de las incongruencias que en ellas se representan. Por un lado, tenemos el test de imagen quimérica, que consiste en la percepción e identificación de un dibujo en el que se representan dos mitades de sendos animales (la mitad izquierda corresponde a un pez y la derecha a un cerdo, en perspectiva lateral) y que se presenta al sujeto durante dos segundos de exposición. Ante este conflicto perceptivo-cognitivo, el sujeto puede responder que realmente se trata de una imagen extraña compuesta por las figuras parciales de cada animal, o puede despreciar uno de los dos componentes para resolver el conflicto (hemiinatención visual), para descubrir la complejidad del conjunto sólo cuando se le muestran luego por separado cada una de las dos mitades. Por otro lado, contamos con la escena visual incongruente, que consiste en la comprensión y descripción de una lámina de la medida de inteligencia de Terman y Merrill (lámina M-VII3a) [23], en la que está representada una acción: un hombre está cortando la rama de un árbol sobre la que está sentado distalmente al punto de corte, por lo que caerá al suelo junto con la rama cuando termine de cortarla. Hemos estudiado la validez de estas dos pruebas comparando las respuestas en tres grandes grupos de sujetos: con TAP, con TDAH y controles. Ambas pruebas poseen una efectividad 
alta y una eficiencia moderada. Los escolares con TAP tienen una capacidad menor para comprender la quimera y/o la incongruencia de la escena compleja que se les muestra. En el análisis discriminante, cerca del $90 \%$ de sujetos queda correctamente clasificado como TAP/no TAP a través de estas pruebas de coherencia central [22].

\section{Conclusiones}

La evaluación de la función de coherencia central es factible en el ámbito clínico mediante pruebas sencillas que han formado parte de escalas psicométricas clásicas. Interpretadas en el contexto del TAP, contribuyen a comprender mejor las dificultades adaptativas de los sujetos afectos.

Bibliografía

1. Crespo-Eguílaz N, Narbona J. Trastorno de aprendizaje procedimental: características neuropsicológicas. Rev Neurol 2009; 49: 409-16.

2. Gillberg C. Deficits in attention, motor control, and perception: a brief review. Arch Dis Child 2003; 88: 904-10.

3. Nava G, Valdez P. Hemi-inatención en niños. Anales de Psicología 2006; 22: 113-9.

4. Blázquez-Alisente JL. Atención y funcionamiento ejecutivo en la rehabilitación neuropsicológica de los procesos visuoespaciales. Rev Neurol 2004; 38: 487-95.

5. Frith U. Autism: explaining the enigma. Oxford: Basil Blackwell; 1989.

6. López B, Leekam S. Teoría de la coherencia central: una revisión de los supuestos teóricos. Infancia y Aprendizaje 2007; 30: 439-57.

7. Witkin HA, Moore CA, Goodenough DR, Cox PW. Field dependent and field independent cognitive styles and their educational implications. Review of Educational Research 1977; 47: 1-64.

8. Norman D, Shallice T. Attention to action: willed and automatic control of behavior. In Davidson RJ, Schwartz GE, Shapiro D, eds. Consciousness and self-regulations. Advances in research and theory. Vol. 4. New York: Plenum Press; 1986. p. 1-18.

9. Colby C. The neuroanatomy and neurophysiology of attention. J Child Neurol 1991; 6 (Suppl): S90-118.
10. Ungerleider LG, Mishkin M. Two cortical visual systems. In Ingle D, Goodale M, Mansfield R, eds. Analysis of visual behavior. Cambridge, Massachusetts: MIT Press; 1982 p. 549-86.

11. Atkinson J, Braddick O. Visual and visuocognitive development in children born very prematurely. In Von Hofsten C, Rosander K, eds. Progress in brain research. Vol. 164. Amsterdam: Elsevier; 2007. p. 123-49.

12. Fazzi E, Bova S, Giovenzana A, Signorini S, Uggetti C, Bianchi P. Cognitive visual dysfunctions in preterm children with periventricular leukomalacia. Dev Med Child Neurol 2009; 51: 974-81.

13. Happé F, Frith U. The weak coherence account: detail-focused cognitive style in autism spectrum disorders. J Autism Dev Disord 2006; 1: 5-25.

14. Van Lang N, Bouma A, Sytema S, Kraijer D, Minderaa R. A comparison of central coherence skills between adolescents with an intellectual disability with and without comorbid autism spectrum disorder. Res Dev Disabil 2006; 27: 217-26.

15. Noens I, Berckelaer-Onnes I. The central coherence account of autism revisited: evidence from the ComFor study. Res Autism Spectr Disord 2008; 2: 209-22.

16. Le Sourn-Bissaoui S, Caillies S, Gierski F, Motte J. Ambiguity detection in adolescents with Asperger syndrome: is central coherence or theory of mind impaired. Res Autism Spectr Disord 2011; 5: 648-56.

17. Attwood T. The complete guide to Asperger's syndrome. London: Jessica Kingsley Publishers; 2006.

18. Happé F. Current psychological theories of autism: the 'theory of mind' account and rival theories. J Child Psychol Psychiatry 1994; 35: 215-29.

19. Booth R, Happé F. 'Hunting with a knife and ... fork': examining central coherence in autism, attention deficit/ hyperactivity disorder, and typical development with a linguistic task. J Exp Child Psychol 2010; 107: 377-93.

20. Jolliffe T, Baron-Cohen S. A test of central coherence theory: linguistic processing in high-functioning adults with autism or Asperger syndrome: is local coherence impaired? Cognition 1999; 71: 149-85.

21. Gillberg C. Developmental and neuropsychiatric disorders of childhood. In Aicardi J, Bax M, Gillberg C, eds. Diseases of the nervous system in childhood. 3 ed. London: Mac Keith Press; 2009. p. 889-901.

22. Crespo-Eguílaz N, Magallón N, Narbona J, SánchezCarpintero R. Percatación de incongruencias en tareas de percepción visual simultánea: estudio de la disfunción neurocognitiva en el trastorno de aprendizaje procedimental [abstract]. In Actas del VIII Congreso Nacional de la Sociedad Española de Neurología Pediátrica. Córdoba, España, 2010.

23. Terman L, Merrill MA. Medida de inteligencia. Madrid: Espasa Calpe; 1976.

\section{Difficulties in the swift recognition of contradictions in procedural learning disorder: possible central coherence dysfunction}

Introduction. Procedural learning disorder, or non-verbal learning disorder, affects the automation of perceptual motor and cognitive skills and routines.

Aim. To further our knowledge of the neurocognitive dysfunction in this disorder and, more specifically, to analyse the difficulties experienced when going from recognition of the constituting elements of something to an understanding of the whole.

Development. Contrasting simultaneous visual information and the swift recognition of contradictions are especially difficult abilities for children with procedural learning disorder.

Conclusions. These difficulties may reflect a central coherence dysfunction and can partly account for the deficient ability to adapt their social behaviour displayed by these children.

Key words. Attention deficit. Central coherence. Non-verbal learning. Procedural learning. Recognition of contradictions. Visuospatial integration. 